\title{
EFFETS RÉSULTANT DE L'ADJONCTION DE GEL D'ALUMINE AU VACCIN CONTRE
} LA PESTE BOVINE

\author{
par H. JACOTOT
}

Par un ensembla de travaux dont on ne saurait trop souligner l'originalité Ja précision, la portée, G. Ramon a fixé la technique et établi la théorie d'une móthode qui permet d'accroître notablement et parfois considérablement les effets d'un grand nombre d'antigènes divers. Lue procédé consiste en principe à adjoindre à l'antigène, toxine ou corps microbiens, une substance de nature à retarder sa résorption et à stimuler dans l'organisme la formation de l'anticorps correspondant.

Entre les mains de RaMon le tapioca et la lanoline ont donné des résultatis surprenants, le premier dans la préparation du sérum antitétanique notamment, la seconde dans l'emploi de l'anatoxine tétanique par exemple. Des effets également significatifs bien que moins accusés ont été otbenus par adjonction de lanoline encore, de saponine, d'alun, de cholestérol, de jaune d'ouf, etc., à des antigènes divers par RAMoN lui-même et par d'autres auteurs.

Il est permis de penser que le gel d'alumine, utilisé pour la première fois par Schmid en 1935 , renforce par un mécanisme analogue le vaccin antiaphteux préparé selon le procédé de Waldmann. Pour autant qu'on l'envisage lorsqu'il a perdu sa virulence, état sous lequel il semble bien que son emploi se soit généralisé, ce vaccin est une émulsion tissulaire formolée du type de celle que VALLÉE et CARRÉ ont préconisée dès 1925 , renforcée par addition d'hydroxyde d'aluminium.

Partant de cette hypothèse je me suis proposé de rechercher si l'addition de gel d'alumine au vacein organique formolé en usage contre la peste bovine permettrait d'améliorer les effets de ce vaccin dans une mesure intéressante. Les premiers résultats de cette étude ont été préciśs en 1940 (1); voici, succinc. tement présentée, la relation de l'ensemble des recherches.

Préparation du mélange vaccin-hydroxyde d'aluminium. Le vaccin constitué par des pulpes organiques finement broyées et émulsionnées dans de l'eau formolée est additionné, quelques jours après sa préparation, alors qu'aucune trace de virus vivant ne peut y être décelée, d'une certaine quantité de gel d'alumine ; on le conservera dans les mêmes conditions qu'un échantillon d'émulsion non additionnée de gel, mais complétée au même volume et qui servira de vaccin témoin.

L'examen au microscope montre que les granules d'hydroxyde d'aluminium se fixent sur les particules de tissus qui constituent le principe actif du vaccin ou plutôt Jui servent de support; ils forment un revêtement plus ou moins

(1) C. R. Acad. des Sciences, 1940, CCX, 376. 
complet autour de chacune d'elles. Lies amas cellulaires de petites dimensions et les cellules libres se réunissent entre eux ou se soudent à des particules plus grosses. T'adsorbat še dépose rapidement; une légère agitation permet de reconstituer la suspension.

Action de l'adjúvant sur l'activité du vaccin frais. - Dans les jours qui suivent la préparation des émulsions, c'est-à-dire lorsque celles-ci n'ont pas subi l'action du vieillissement, le vaccin ádsorbé sur le gel d'alumine se montre au moins dix fois plus actif que le vaccin témoin; à partir d'une certaine dose, variable d'un śchantillon à l'autre, il exerce une action très régulière.

Expériences. - a) Il s'agit d'émulsions vaccinales préparées quelques jour's ávant. Cinq veanx reçoivent respectivement, un quart, un demi, un, deux et quatre centimètres-cubes de vacein adsorbé, cinq autres veaux reçoivent respectivement un et demi, trois, cinq, huit et douze centimètres-cubes de vaccin témoin. On les éprouve tous, quinze jours après, par in oculation de 2 ce. de sang virulent. Parmi les premiers, celui qui a reç 0 cc. 25 de vaccin présente une immunité partielle, les quatre autres une immunité totale; parmi les seconds ceux qui ont reçu un et demi, trois et einq centimètres-cubes de vaccin ne bénéficient d'aucune immunité, celui qui a reçu 8 cc. offre une résistance partielle; seul celui qui a reçu 12 cc. présente uné immunité totale; le rapport d'activité des deux vaccins est de $1 / 25$ environ.

b) Il s'agit encore de vaccins qui ont été préparés. quelques jours avant. Quatre veaux reçoivent respectivement un demi, un, deux et quatre centimètres cubes de vaccin adsorbé; quatre autres veaux reçoivent un centimètre-cube et demi," trois, six et neuf centimètrés-cubes de vaccin témoin. A l'épreuve parmi les premiers, celui qui a reçu un demi-centimètre-cube de vaccin adsórbé offre une résistance presque entière, les autres une résistance parfaite; parmi les seconds ceux qui ont reçu un et demi et six centimètres-cubes de vaccin contractent une peste grave, celui qui a reçu trois centimètres-cubes offre une résistance presque entière, le dernier ane résistance parfaite.

Effets de l'adjuvant sur la conservation du vaccin. - L'étude d'un grand nombre d'émulsions vaccinales conservées ou à la température du laboratoire (25 à $\left.30^{\circ}\right)$ ou à la glacière (5 à 100) a permis de les préciser. De l'ensemble des observations, il ressort que le vaccin adsorbé conserve son activité beaucoup plus longtemps que le vaccin témoin; c'est ainsi que leur rapport d'activité étant 1/10, 1/15 ou 1/20 immédiatement après la préparation est 1/20,1/30 ou 1/40 deux ou trois mois plus tard lorsqu'on les a conservés l'un et l'autre en milieu chaud' et quatre ou cinq mois lorsqu'on les a conservés en milieu froid.

Au surplus, en vieillissant, le vaccin adsorbé conserve une régularité d'action que le vaccin témoin ne présente pas toujours quand il est frais et dont il est généralement dépourvu après peu de temps; le vaccin adsorbé ne commence à présenter des défaillances qu'après six ou huit mois de séjoúr en milieu chaud et dix ou douze mois en milieu froid.

Expérienees. - a) Il s'agit d'émulsiens. vaccinales qui ont été conservées pendant deux mois dans des conditions particulièrement défavorables : fraîches, 
elles engendraient une immunité totale aux doses respectives de 1 cc. (vaccin adsorbé) et $9 \mathrm{cc}$. (vaccin témoin). Cinq veaux reçoivent respectivement un demi, un, deux, quatre, huit centimètres-cubes de vaccin adsorbé, et cinq autres veaux, trois, six, dix, quinze, vingt-cinq centimètres-cubes de vaccin témoin. A l'épreuve virulente, parmi les premiers, ceux qui ont reçu 0 cc. 5 et 1 cc. de vaccin présentent une immunité partielle, les autres une immunité totale; parmi les seconds tous contractent une peste caractérisée; le dernier (25 cc.) offre une lègère résistance; le rapport d'activité des deux vaccins qui était originellement de 1,9, est compris, deux mois après, entre $1 / 25$ et $1 / 50$.

b) Il s'agit d'émulsions vaccinales qui ont été conservées pendant quatre mois à la température de $8^{\circ}$ puis, pendant deux mois, à la température de 320 . Trois veaux reçoivent uniformément 5 cc. de vaccin adborbé et trois 5 cc. de vaccin témoin. On les éprouve 15 jours après. Les trois premiers opposent une résistance totale à l'inoculation virulente, les trois autres contractent une peste grave quoique non mortelle.

Influence de l'adjuvant sur le temps d'établissement de I'immunité. - Plusieurs expériences ont été faites consistant à éprouver simultanément des veaux qui avaient été vaccinés 12 jours, 7 jours et 3 jours avant, respectivement; chaque expérience comportait deux séries d'animaux dont les uns recevaient le vaccin adsorbé, les autres le vaccin témoin; préalablement, on avait établi le rapport d'activité des deux vaccins et chacun fut ensuite employé à une dose uniforme voisine de la dose liminaire.

La conclusion est que les émulsions tissulaires additionnées de gel d'alumine engendrent l'immunité dans les mêmes délais que les émulsions ordinaires; expérimentalement, les sujets qui ont reçu l'une ou l'autre se montrent totalement résistants à l'égard du virus inoculé entre le cinquième et le dixième jour qui suivent la vaccination.

Influence de I'adjuvant sur la persistance de l'immunité. Des essais effectués on peut conclure que, à l'égard du virus inoculé, l'immunité engendrée par une seule dose de vaccin au gel d'alumine persiste plus longtemps que l'immunité engendrée par une seule dose de vaccin ordinaire; dans le premier cas elle dépasse assez souvent huit semaines; dans le second elle n'atteint pas toujours six semaines. Il est permis de penser que vis-à-vis de la contamination cet écart s'accuserait; la pratique des vaccinations semble le confirmer.

Expériences. - On fait usage d'émulsions vaccinales qui ont été préparées un mois avant; huit veaux reçoivent 1 ec. 5 du vaccin adsorbé, dose voisine de la dose liminaire, huit veaux reçoivent 15 ce. de vaccin témoin, quantité également voisine de la dose liminaire. Les résultats des inoculations d'épreuve sont exprimés conformément à la convention suivante :

$0=$ aucun signe morbide $;+=$ réaction très légère $++=$ réaction légère; $+++=$ réaction d'intensité moyenne; $++++=$ maladie grave. 
Vacein au gel $\begin{array}{cc}\text { Veau, } 31,0 \\ -\quad 40 & 0\end{array}$

Veau $33: 0$
Epreuve après 3 semaines $\left\{\begin{array}{ccc}\text { Veau } & 41 & 0 \\ - & 45 & +\end{array}\right.$

Epreuve après 6 semaines $\left\{\begin{array}{l}\text { Veau } 44++ \\ -42+++\end{array}\right.$

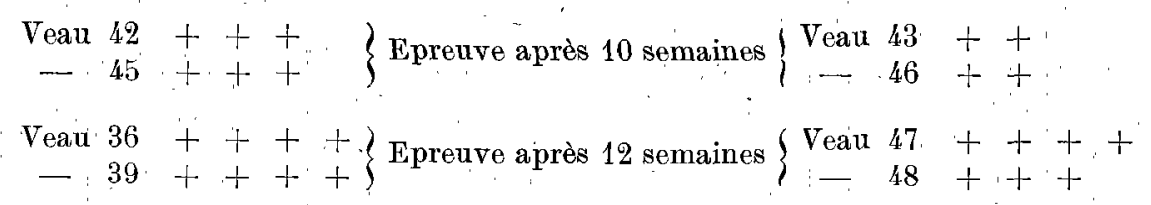

Teneur en gel d'alumine et pouvoir immunigène du vaccin. Dańs le cadre des essais, l'activité du varcin s'est montrée en rapport direct avec la quantité d'hydroxyde d'aluminium qu'il contenait, les pulpes additionnées de $75 \%$ d'adjuvant ayant un pouvoir immunigène plus élevé que célles additionnées de $50 \%$, plus élevé éncore que celles additionnées de $25 \%$ de gel.

Dans la pratique, le táux de l'adsorbant est fixé en considération de certaines nécessités qu'il faut concilier : augmenter l'activité du vaccin, ne pas donner à l'émulsion une consistance trop épaisse, conserver à la dose utilitaire un volume réduit.

Le vaccin antipestique délivré par l'Institut Pasteur de -Nhătrang est à base d'amygdales, ganglions, rates et poumons; on incorpore aux pulpes $60 \%$ de gel d"alumine titrant $5,5 \mathrm{~d}^{\prime} \mathrm{A}^{2} \mathrm{LO}^{3} 3 \mathrm{H}^{2} \mathrm{O}$; l'émulión finale est au $1 / 5$ et contient $5,5 \%$ de formol.'

Mode d'action du: gel d'alumine. ${ }^{*}$ Le gel d'alumine paraît exercer un double rôle : par. sa résistance à la résorption et son union intime à la substance du vaccin, il stimule l'élaboration de l'immunité (action sur l'organisme); et 'd'autre part, il préserve les émulsions, formolées de l'affaiblissement (action sur le vaccin) en neutralisant; c'est probable, par un mécanisme indéterminé, l'excès d'aldéhyde formique.

Avantages techniques et pratiques de l'emploi du vaccin au gel d'alumine. - De 1930 à 1939, l'Institut Pasteur de Nhatrang a mis à la disposition du Service des épizooties d'Indochine un vaccin formolé et un vacein toluéné non additionnés. d'adjuvants; 61 millions de centimètres cubes en ont été délivrés. Le vaccin formolé peut être préparé très rapidément en raison du pouvoir microbicide de l'aldéhyde formique, mais il s'affaiblit rapidement. Le vaccin toluéné vaccine plus fortement et plus régulièrement, la résistance qu'il engendre est plụs durable et il ne s'affaiblit que lentement; mais sa préparation est longue 
en ce sens que la stérilisation des germes adventices s'effectue lentement dans les émulsions tissulaires soumises à l'action du toluène.

Le vaccin au gel d'alumine réunit les avantages de l'un et l'autre des précédents ; sa supériorité résulte de ce qu'il possède trois qualités primordiales : $1^{0}$ sa préparation est rapide parce qu'il est un vaccin formolé; $2^{\circ}$ il possède, quelques jours après la préparation, un pouvoìr immunigène très supérieur' à celui des émulsions ordinaires, agissant à dose dix fois moindre que le vaccin formolé et cinq fois moindre que le vaccin toluéné; $3^{\circ}$, il conserve une haute et franche activité beaucoup plus longtemps que les vaccins ordinaires, les vaccins formolés notamment.

Indépendamment de leurs incidences techniques, ecs faits ont des conséquences pratiques importantes : $a$ ) possédant un pouvoir élevé, le vaccin au gel peut être employé à des doses inférieures à celles des vaccins ordinaires d'où, pour le personnel chargé des vaccinations un gain de temps et une économie de matériel $b$ ) la réduction du volume de la dose, de sa teneur en parenchyme, réduction corrélative d'une plus grande activité des émulsions, entraîne une diminution des dépenses de fabrication et de stockage en chambre froide; $c$ ) le vaccin ne s'affaiblissant que lentement on peut en constituer des réserves, d'où plus de régularité dans la fabrication, d'aisance dans les livraisons, de souplesse dans l'utilisation; $d$ ) enfin et c'est probablement l'avantage le plus précieux, l'emploi du gel d'alumine en tant qu'adjuvant du vaccin antipestique permet de réduire dans une proportion importante, presque de trois à un le nombre d'animaux nécessaires à la fabrication.

De 1940 à 1945, il a été employé en Indochine 55 millions de centimètres-cubes de vacein antipestique àu gel d'alumine et l'on peut évaluer à deux millions le total des animaux vaccinés. Partout où elle a été judicieusement et correctement appliquée la vaccination par pulpes organiques adsorbées a conduit aux résultats attendus.

INSTITUT PASTEUR DE. NHATRANG.

(Indochine) 